\title{
A Large-Core Compact High-Power Single-Mode Photonic Crystal Fiber Laser
}

\author{
Arash Mafi, Jerome V. Moloney, Dmitri Kouznetsov, Axel Schülzgen, Shibin Jiang, Tao Luo, and \\ Nasser Peyghambarian
}

\begin{abstract}
A novel method for making a compact high-power single-mode photonic crystal fiber laser (PCFL) is proposed. The entire photonic crystal fiber is doped uniformly with active material yet only the core is pumped. Numerical simulations predict that, by using Er-Yb codoped phosphate glass, it is possible to extract 9.5 W from a 1.8-cm-long PCFL with a 100- $\mu$ m core diameter pumped with $20 \mathrm{~W}$, achieving near $50 \%$ efficiency.
\end{abstract} tals.

\section{INTRODUCTION}

H IGH-POWER fiber lasers have recently been the subject of intense research [1]-[7]. Good beam quality, robustness, and relative ease of manufacturing are important characteristics of these lasers. There have been reports of extracting a few hundred watts of power from single-mode and multimode double-clad fiber lasers [1], [2]. However, these fibers are typically a few meters long. Shorter fiber lasers offer the possibility of achieving high-power single-mode single-frequency operation while mitigating against detrimental nonlinear optical effects such as simulated Brillouin scattering (SBS) and stimulated Raman scattering (SRS). Generating high output power from a short fiber still remains a challenge since it requires large pump absorption and high signal gain per unit length of the fiber. Both can theoretically be achieved by heavily doping the core of the fiber with active material and/or choosing a large core. However, it is well known that high concentrations of active rare-earth dopants can have a negative impact on the efficiency of the laser. In Er-doped silica fibers, high concentration results in serious degradation of gain and pump efficiency induced by stepwise and cooperative upconversion. Some of us have shown that higher ion concentration without clustering effects can be achieved by using phosphate glass as host [8]. Er-Yb codoping also broadens the absorption band and increases the pump absorption by two orders of magnitude. The pump energy that is absorbed by $\mathrm{Yb}$ ions is quickly transfered to neighboring $\mathrm{Er}$

Manuscript received December 11, 2003; revised July 20, 2004. This work was supported by the Air Force Office for Scientific Research, Air Force Material Command, USAF, under Grant F49620-02-1-0380.

A. Mafi and J. V. Moloney are with the Optical Sciences Center, University of Arizona, Tucson, AZ 85721 USA, and also with the Arizona Center for Mathematical Sciences, University of Arizona, Tucson, AZ 85721 USA (e-mail: mafi@acms.arizona.edu).

D. Kouznetsov is with the Arizona Center for Mathematical Sciences, University of Arizona, Tucson, AZ 85721 USA.

A. Schülzgen and N. Peyghambarian are with the Optical Sciences Center, University of Arizona, Tucson, AZ 85721 USA.

S. Jiang and T. Luo are with NP Photonics, Tucson, AZ 85747 USA.

Digital Object Identifier 10.1109/LPT.2004.836749 ions in the ground level, exciting them to the pump level from where they rapidly relax to the metastable level. The nonradiative relaxation rate into the metastable level in $\mathrm{Er}$ ions is large in a phosphate glass host resulting in a negligible backtransfer from the Er pump level to the Yb ground level, thus increasing the pumping efficiency. Considering all the advantages of the Er-Yb codoped phosphate glasses, in our analysis, we use a phosphate glass that is uniformly doped with $1 \mathrm{wt} \% \mathrm{Er} 2 \mathrm{O} 3$, $2 \mathrm{wt} \% \mathrm{Yb} 2 \mathrm{O} 3$ and is manufactured by NP Photonics [8].

Increasing the core size of a conventional single-mode fiber brings its own difficulties. The required step in the refractive index between the core and cladding scales quadratically with the inverse of the core diameter. Even with extremely fine index tuning of the order of $\Delta n \sim 10^{-4}$, it is not possible to design a regular single-mode step-index fiber with a core diameter larger than $\sim 60 \mu \mathrm{m}$ for $n \sim 1.5$ at $\lambda_{s}=1535 \mathrm{~nm}$. This problem can be overcome by using the novel concept of photonic crystal fiber (PCF) confinement [9], [10]. Several papers have suggested lasers based on a PCF where only the core of the PCF is active and step index is fine tuned to closely match that of the cladding. In [3]-[6], the desired step in the index of refraction is achieved by controlling the refractive index of the core by choosing appropriate glass host and dopants. Alternatively, [7] suggests a core constructed from a combination of many doped and undoped spots. The effective index of refraction of the core can be controlled by the ratio of the area of doped spots to that of the undoped. The collection of these doped and undoped spots creates an effective refractive index that is close enough to that of the cladding. However, [7] reports that only $10 \%$ of the total area of the core is doped which is not very efficient. None of the solutions above genuinely employs the novel concept of PCF and can all be applied to regular double-clad fiber lasers. The real power of PCF resides in the fact that the entire PCF can be made of the same material with a uniform refractive index. The effective index of refraction of the cladding is solely reduced by introducing holes into the fiber where the radius of the holes can accurately fine tune the desired effective index step.

\section{VIABLE PCF LASER (PCFL)}

In this letter, we numerically model a novel PCFL that is made entirely from an active material and is pumped only in the core. This geometry has the advantage that a step index is not introduced between the cladding and the core. The centers of the airholes running along the PCF are located on a triangular lattice of pitch size $\Lambda=50 \mu \mathrm{m}$ as in Fig. 1. A missing airhole in the center of the PCF forms a guiding core with an approximate 


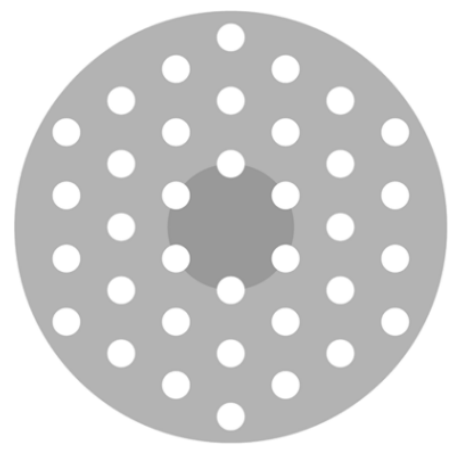

Fig. 1. PCF made entirely from an Er-Yb codoped phosphate glass. The core is marked by a darker color for illustration purposes only.

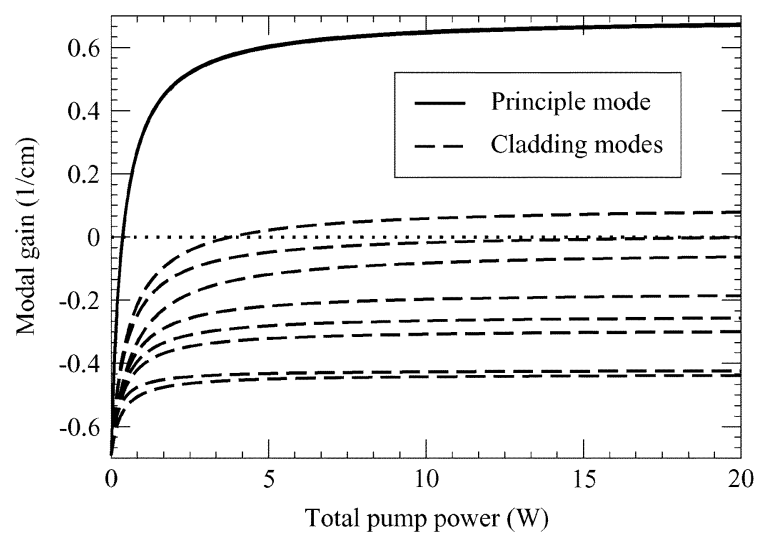

Fig. 2. Values of the gain of the principle mode and the next eight highest gain cladding modes as a function of the total pumping power.

diameter of $2 \Lambda \sim 100 \mu \mathrm{m}$. The area surrounding the core is referred to as the cladding and has a slightly lower effective index of refraction due to the presence of the airholes. The overall diameter of the structure is determined by the number of airhole layers in the cladding. In our design, we analyze a PCF with three layers of airholes, as shown in Fig. 1, adequate to confine the pump and signal in the core region. The ratio of the diameter of the airholes to the pitch size is 0.36 , making the PCF single mode at $\lambda_{s}=1535 \mathrm{~nm}$. There is only one mode that resides almost entirely in the core. There are, however, numerous other modes exhibiting small overlap with the core that could be potentially excited in the cladding but these are strongly absorbing. The gain values of the principle mode and the next eight highest gain cladding modes are plotted in Fig. 2. The horizontal axis represents the total pump power that is assumed to be uniformly distributed in the core. For simplicity, the signal power is taken to be zero. From Fig. 2, one can conclude that when signal saturation and various loss mechanisms are also taken into account, the principle mode is the only mode with large enough gain and all the cladding modes are strongly absorbed by the cladding during multiple passes. Based on these observations, we are ensured that the PCF will truly work as a single-mode fiber laser.

\section{DESIGNING THE OPTIMUM LASER CAVITY}

We now consider the design of the laser cavity including the appropriate length of the PCF and reflectivities of the mirrors for the maximum output signal power. Ideally, we need a single-mode pump source with $100-\mu \mathrm{m}$ diameter and NA =
0.01 (the NA of the PCF) to maximally couple and confine the pump light in the core of the PCF. Commercially available high-power multimode sources are not suitable because of their large NA. We ran a simulation using a beam propagation method (BPM) code and pumped the PCFL in the core using a $100-\mu \mathrm{m}$ diameter multimode laser with NA $=0.2$. We observed that within the first few millimeters of propagation, a large portion the pump modes, especially those with large incidence angles, leaked into the cladding and were not absorbed by the core. However, we emphasize that the PCFL does not necessarily require an idealized pump source. For example, our BPM simulation shows that a single-mode pump source with $350-\mu \mathrm{m}^{2}$ mode-area and NA around 0.05 has a $77 \%$ absorption efficiency compared to an ideal pump. [11] recently demonstrated a near-diffraction limited $30-\mathrm{W}$ optically pumped semiconductor laser at $980 \mathrm{~nm}$. The $500-\mu \mathrm{m}$ diameter output can be focused to $100 \mu \mathrm{m}$ and NA $<0.01$ using an optical telescope making it a near ideal pump source for our PCFL. We note that unless pumped very hard, with significant leakage into the cladding, high threshold cladding modes will not be excited. Since large reflectivities are required for efficient lasing of our PCFL, we may discriminate against potential cladding modes by coupling end mirrors only to the core of the PCF (for example butt coupling a $100-\mu \mathrm{m}$ diameter fiber, coated with a mirror). Although the PCF is strongly single-mode for the signal at $\lambda_{s}=1535 \mathrm{~nm}$, it is quite forgiving for the pump at $\lambda=976 \mathrm{~nm}$. The reason is that the core of the PCF is in general more confining at shorter wavelengths. In addition, the signal goes through multiple passes in the PCF allowing higher order modes to leak out. This is in contrast to the pump that travels only twice along the short PCF before being almost entirely absorbed in the core.

In order to calculate the output signal power, we solve the following set of differential equations:

$$
\begin{aligned}
& \frac{d P_{\text {pump }}^{ \pm}(z)}{d z}=\mp a\left(P_{\text {pump }}^{\text {tot }}, P_{\text {signal }}^{\text {tot }} ; z\right) P_{\text {pump }}^{ \pm}(z) \\
& \frac{d P_{\text {signal }}^{ \pm}(z)}{d z}= \pm g\left(P_{\text {pump }}^{\text {tot }}, P_{\text {signal }}^{\text {tot }} ; z\right) P_{\text {signal }}^{ \pm}(z) .
\end{aligned}
$$

$P_{\text {pump }}^{ \pm}(z)$ and $P_{\text {signal }}^{ \pm}(z)$ are the forward and backward moving pump and signal power. $a$ and $g$ are the effective pump absorption and signal gain factors as a function of the total signal and pump power, respectively. In order to calculate $a$ and $g$ based on the particular transverse profiles of the signal and power in the PCF, we use a full vectorial Maxwell solver based on the finite-element method (FEM). The FEM code solves for the transverse profile, propagation constant, and the effective gain of the modes, especially the desired principle mode. The transverse profile of the complex refractive index is used in the FEM code to carry out these calculations at a particular frequency. In turn, the imaginary part of the refractive index is calculated using the signal and pump intensities and the kinetic equations for the population of the lasing levels in the Er-Yb system, following [12]. It is possible to converge to a consistent solution using this method by an appropriate initial guess and a few iterations.

For maximum output power, the input mirror should have $100 \%$ transmission at the pump wavelength and $100 \%$ reflectivity at the signal wavelength. Furthermore, the output mirror 


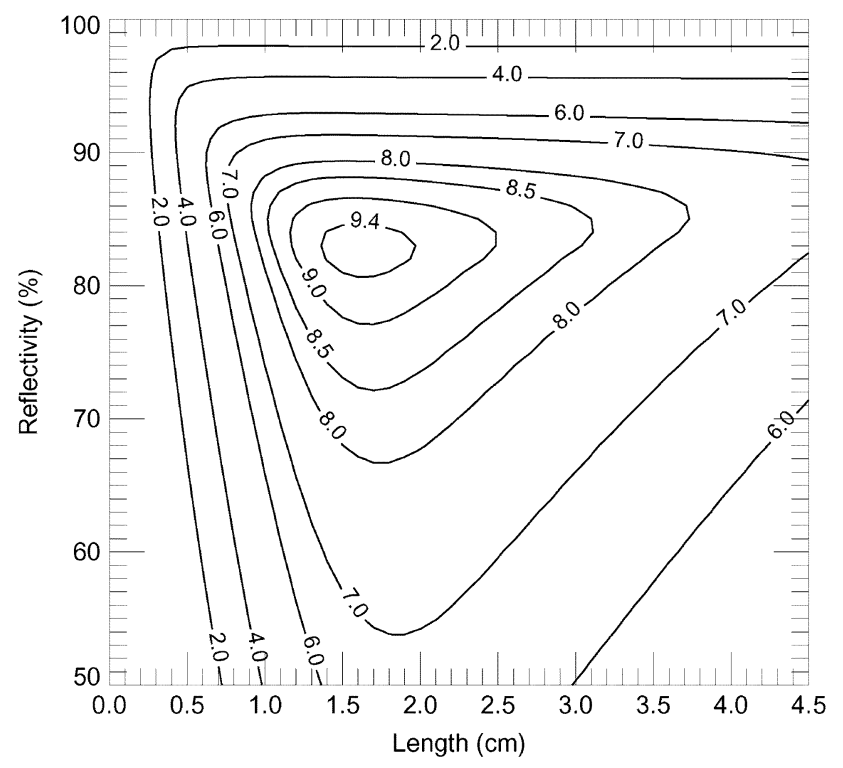

Fig. 3. Output signal power in watts as a function of the length of the PCFL and the reflectivity of the output mirror at the signal wavelength for $20 \mathrm{~W}$ of pump power.

should exhibit $100 \%$ reflectivity at the pump wavelength. With these assumptions, the differential equations are solved iteratively with the appropriate boundary conditions until the solution converges. In Fig. 3, we show a contour plot of the output signal power in watts as a function of the length of the PCF and the reflectivity of the output mirror at the signal wavelength for $20 \mathrm{~W}$ of input pump power. The peak output signal power is almost $9.5 \mathrm{~W}$ and happens at a cavity length around $\sim 1.8 \mathrm{~cm}$ and output mirror reflectivity $83 \%$. The output signal is single-mode with $\mathrm{M}^{2}$ very close to unity. In Fig. 3, we have assumed a uniform pump intensity distribution across the core. An adjustment is required for specific pump profiles. Note that in our calculations, we have not included any loss mechanisms due to the host glass and dopants and also imperfections in the PCF, especially scattering at the glass-airhole boundaries. With increasing loss, the optimum value of the output mirror signal reflectivity decreases. However, these modifications will only have a very small effect on our results since the PCF is quite short.

\section{CONCLUSION}

In this letter, we have numerically analyzed a high-power single-mode PCFL. Unlike the previous work on PCFL that consider an active core with a passive cladding, our PCF is entirely made from an active glass. We are, thus, assured that an unwanted step index would not be created in the core-cladding interface leading to an undesirable multimode fiber. In addition, our single glass approach eases the fabrication of the PCF drastically. The effective size of the core of the PCF is $\sim 100 \mu \mathrm{m}$. The active material is a phosphate glass that is uniformly doped with $1 \mathrm{wt} \% \mathrm{Er} 2 \mathrm{O} 3$ and $2 \mathrm{wt} \% \mathrm{Yb} 2 \mathrm{O} 3$. We have shown that the PCFL is single-mode. The large value of reflectivity of the output mirror required for optimum power results in multiple passes of the signal beam in the cavity. This ensures that the higher order signal modes leak into the surrounding absorbing cladding. The strong absorption of the pump allows us to design a very short yet efficient single-mode laser. The achievable efficiency of near $50 \%$ results in the extraction of a signal output power near $9.5 \mathrm{~W}$ from a 1.8-cm-long laser pumped with $20 \mathrm{~W}$. Nonlinear scattering effects such as SBS and SRS and also amplified spontaneous emission are very small due to the short length of the PCFL. At 20-W pump power, the maximum temperature in the PCF $\left(T<250^{\circ} \mathrm{C}\right)$ is well below the glass transition temperature $\left(T=460^{\circ} \mathrm{C}\right)$. Thermooptical effects may be safely ignored since $d n / d T \sim-5 \times 10^{-7} 1 /{ }^{\circ} \mathrm{C}$ and the fact that the temperature stays nearly uniform throughout the PCFL.

\section{ACKNOWLEDGMENT}

The authors would like to thank P. Kano for providing his BPM code to simulate the pump absorption.

\section{REFERENCES}

[1] J. Nilsson, J. K. Sahu, Y. Jeong, W. A. Clarkson, R. Selvas, A. B. Grudinin, and S. Alam, "High-power fiber lasers: New developments," in Advances in Fiber Devices, L. N. Durvasula, Ed., 2003, pp. 50-59. Proc. SPIE 4974.

[2] J. Limpert, A. Liem, H. Zellmer, and A. Tunnermann, "500 W continuous-wave fiber laser with excellent beam quality," Electron. Lett., vol. 39, pp. 645-647, Apr. 2003.

[3] K. Furusawa, A. N. Malinowski, J. H. Price, T. M. Monro, J. K. Sahu, J. Nilsson, and D. J. Richardson, "Cladding pumped Ytterbium-doped fiber laser with holey inner and outer cladding," Opt. Express, vol. 9, pp. 714-720, 2001.

[4] P. Glas and D. Fisher, "Cladding pumped large-mode-area Nd-doped holey fiber laser," Opt. Express, vol. 10, pp. 286-290, 2002.

[5] J. Limpert, T. Schreiber, S. Nolte, H. Zellmer, T. Tunnermann, R. Iliew, F. Lederer, J. Broeng, G. Vienne, A. Petersson, and C. Jakobsen, "Highpower air-clad large-mode-area photonic crystal fiber laser," Opt. Express, vol. 11, pp. 818-823, 2003.

[6] A. Cucinotta, F. Poli, S. Selleri, L. Vincetti, and M. Zoboli, "Amplification properties of $\mathrm{Er}^{3+}$-doped photonic crystal fibers," J. Lightwave Technol., vol. 21, pp. 782-788, Mar. 2003.

[7] W. J. Wadsworth, R. M. Percival, G. Bouwmans, J. C. Knight, and P. S. J. Russell, "High power air-clad photonic crystal fiber laser," Opt. Express, vol. 11, pp. 48-53, 2003.

[8] S. Jiang, S. Mendes, Y. Hu, G. Nunzi-Conti, A. Chavez-Pirson, Y. Kaneda, T. Luo, S. Hocde, D. T. Nguyen, E. Wright, J. Wang, W. Tian, T. Nikolajsen, and N. Peyghambarian, "Compact multimode pumped erbium-doped phosphate fiber amplifiers," Opt. Eng., vol. 42, pp. 2817-2820, 2003.

[9] J. C. Knight, T. A. Birks, P. St, J. Russell, and D. M. Atkin, "All-silica single-mode optical fiber with photonic crystal cladding," Opt. Lett., vol. 21, pp. 1547-1549, 1996.

[10] - "All-silica single-mode optical fiber with photonic crystal cladding: Errata,” Opt. Lett., vol. 22, pp. 484-485, 1997.

[11] J. Chilla, S. Butterworth, A. Zeitschel, J. Charles, A. Caprara, M. Reed, and L. Spinelli, "Hiph power optically pumped semiconductor lasers," in Solid State Lasers XIII: Technology and Devices, R. Scheps and H. J. Hoffman, Eds., 2004, pp. 143-150. Proc. SPIE 5332.

[12] M. Karasek, "Optimum design of $\mathrm{Er}^{3+}-\mathrm{Yb}^{3+}$ codoped fibers for largesignal high-pump-power applications," IEEE J. Quantum Electron., vol. 33, pp. 1699-1705, Oct. 1997. 